\title{
Intelligent User Assistance for Cost Effective Usage of Mobile Phone
}

\author{
Deepak P, Anuradha Bhamidipaty, Swati Challa \\ IBM Research, India Research Lab, Bangalore \\ \{deepak.s.p, abhamidi, swatichalla\}@in.ibm.com
}

\begin{abstract}
Cost is the governing factor which defines the penetration and adoption of mobile phones in lower strata (lower income group) of the society particularly in developing countries like India. In this paper we describe an enhancement to the mobile phone design which interacts with the user to facilitate cost-conscious usage of the mobile phone. In particular, we propose an intelligent component in the mobile phone which tracks mobile usage pattern and informs the user of deviations in usage and suggests means of using the device cost effectively.
\end{abstract}

\section{Author Keywords}

Mobile Phone Interfaces, Cost-Effective Usage, Pattern Analysis

\section{ACM Classification Keywords}

I.2.6 [Artificial Intelligence]: Learning-knowledge acquisition, parameter learning; H.5.2 [Information Interfaces and Presentation]: User Interfaces evaluation/methodology

\section{INTRODUCTION}

Developing countries like India, China are noticing an increasing trend in the usage of mobile phones. One of the factors driving this growth is the penetration of mobile phones in the lower income groups [1]. Penetration of mobile phones to the lower strata of the society has been shown to be desirable for economic development [5], in addition to closing the rich-poor divide in accessibility to the information and communication infrastructure. Reduced cost of ownership and attractive tariff plans have appealed the people in lower strata to adopt the mobile phone. Despite this, cost is still perceived to be an important deterrent in the widespread usage of the mobile phone in this section of society [2,3]. Our previous study has indicated that people in this group who own mobile phones

Permission to make digital or hard copies of all or part of this work for personal or classroom use is granted without fee provided that copies are not made or distributed for profit or commercial advantage and that copies bear this noti! ce and the full citation on the first page. To copy otherwise,! to repu blish, to post on servers or to redistribute to lists, requires prior specific permission and/or a fee.

IUI'08, January 13-16, 2008, Maspalomas, Canary Islands, Spain. tend to use it minimally to keep cost in control and find ways of achieving "cheap-usage" of the mobile phone [4].

Users in lower income groups typically confine to a fixed monthly budget for mobile phone expense and try to keep their usage cost within that amount. They manually analyze their day-to-day expense and rely on their instinct to decide the duration of a call and when to avoid or make calls. Practicing cost check manually is unavoidable for the lower income group; but it is very error-prone and often time leads to overshooting the budget and having to accommodate extra cost in the month.

In this paper, we present techniques to learn from the mobile usage pattern and assist the user in being costconscious thus enabling the mobile device do pro-active cost control. Towards that end, we describe an intelligent component in the mobile phone that analyzes the mobile usage pattern and provides proactive alerts and suggestions to check cost usage and control call durations. This would prevent the user from incurring cost that is not aligned with their allocated budget or spending capacity. In the next section we discuss what constitutes cost effective mobile usage for the lower income group and thereafter present the design for the component that automates the mobile usage cost control.

\section{COST EFFECTIVE MOBILE USAGE}

The lower income groups in developing countries like India and China live on a very low income ranging from $1-2 \$$ per day in the rural areas and 5-6\$ per day in the urban areas. The mobile usage cost typically is in the range of $3-15 \$$ per month [4] which is a significant fraction of the income (that is left after all the living costs). We did a study of usage cost optimizations practiced by people in the lower income group by talking to a group of 20-30 people in the said category in the city of Bangalore in India. We found that they optimize usage costs using the following methods:

- Many mobile service providers provide various features that could be optimized on. Certain features provided by Indian providers include free calls to customers of the same provider, reduced rates for calls to friends and family etc. It is very common for the entire family and the close circle of friends to use connections from the same service provider to leverage those offers. 
- Cheap alternatives are often chosen rather than actually making a phone call. Such alternatives include sending an SMS (which is usually cheaper) or making a missed-call to convey agreed-upon Boolean information.

- Manually keeping track of the mobile usage cost incurred, and making sure that calls are scheduled, made and disconnected at such times so that the usage cost is held within the budget.

The focus of this paper is on the third aspect. We directed the user survey on identifying what efforts are being undertaken manually to optimize mobile usage cost and to derive insights to decide on how an intelligent learning component that seeks to automate the manual process should work. The survey led to two interesting findings which could be enumerated as follows:

- Most mobile users in the lower income group have a regular and a largely predictable schedule at home and at workplace. This reflects in their mobile usage too, in that they usually make predecided calls and messages and the recipients (of the call or message) are similar sets of people across days. This leads to a strong pattern of cost incurring mobile usage activities that recurs (mostly) on a daily basis for most people, although certain people have different patterns for workdays and weekends.

- $\quad$ The manual task of restricting mobile usage cost to fall within the budget is done on a best-effort basis and they end up overshooting the budget very frequently as the manual process is very error prone and hard to manage.

Drawing cues from these observations, we present a learning component in the next section that relies on the patterns of mobile usage and proactively gives suggestions on scheduling activities and deciding durations of activities. It may be noted that this component is not specific to the lower income group, but would benefit any mobile user who follows a regular usage pattern and would like to automate usage cost control.

\section{AUTOMATING USAGE COST CONTROL}

In this section, we describe our approach of automating mobile usage cost control in cases where the user follows a recurring pattern of mobile usage. The approach uses two modules; a "discovery module" that discovers the pattern and a "suggestion generator module" which gives proactive suggestions to aid cost control.

\section{Patterns in Mobile Usage}

A pattern is a set (unordered) of types of cost incurring mobile usage activities (referred to as simply calls hereafter), each type tagged with a frequency of occurrence. There would also be a duration associated with the pattern (which could be day, week etc). A usage log is said to contain a pattern if the set of entries corresponding to each duration instance (day, week etc) corresponds to the frequencies as in the pattern. It may be noted that there is no ordering assumed in a pattern. An algorithm for pattern discovery would have to be tolerant to random fluctuations due to noise. We present such an algorithm in the succeeding section.

\section{Discovering Patterns from Usage Histories}

The usage history $\log$ is a sequence of entries ordered in time. Each entry in the log includes: type/duration/time of activity and category of recipient (friend, relative, workrelated etc.). Activities that we consider here are costincurring ones like making outgoing calls and sending short messages (SMS). The usage history $\log$ is input to a clustering algorithm which clusters mobile usage activities of similar nature together. A recurring distribution of activities with certain duration across these clusters would identify a mobile usage pattern. Pattern duration is highlydependent on the usage and is very user-specific.

We determine the mobile usage pattern using the algorithm in Figure 1.

$\mathrm{CD}$ is the set of candidate durations.

- For each entry $\mathrm{cd}_{\mathrm{i}}$ in $\mathrm{CD}$, split the log into segments of the duration. Each segment will have a distribution of activities of varied nature along with their frequencies.

- Compute the similarity of these segments by calculating the aggregated entropy of the distribution of each type of activity across the segments. The lesser the entropy, the more uniform is the presence of each activity type across the segments, which in turn implies that the sets are more similar.

Choose the duration which gives the least entropy below a threshold. Output the pattern corresponding to this duration as the average of split logs (bags of nature of activities) which gives the mean distribution of calls.

If for no duration the entropy is below a threshold, report the non-existence of patterns.

Figure 1. Pattern Discovery Algorithm

\section{Generating Real-Time Suggestions}

The suggestion generator module generates suggestions to enable semi-automatic usage cost control. There are two ways in which the user may deviate from the pattern; either by not making enough calls of a specific type, or by making more calls of a specific type. Cost control being the motive, the suggestion generator needs to generate alerts only if the deviation of the latter kind happens as the former deviation does not result in increased usage cost. For every activity (making a voice call or sending a short message), the suggestion generator module intercepts the request and gives either of the following suggestions:

- Reconsider decision to make the call, 
- Preset the call duration to a suggested duration,

- Use an alternative mechanism than the one attempted (such as "send an SMS instead of making a voice call”) OR

- None

The first three are suggestions which would enable restricting the user to his usual pattern. The suggestion generator module uses the algorithm in Figure 2.

When a call is attempted (called the current activity) on the mobile phone:

- Get the history, i.e., list of activities already performed by the user in the current instance of the duration (day, week etc) corresponding to the pattern discovered.

- Compare the current activity and the history against the pattern. Note that the information on current activity would not have the duration since the activity is not yet performed by the user.

- Given the history, if the current activity does not confirm the pattern i.e., performing the current activity would result in a deviation to the pattern (beyond a threshold), report an alert if the user would like to reconsider the decision to perform the activity.

- If the current activity conforms to the pattern, proactively suggest the likely duration of the call so that the adherence to the pattern is maximized.

- If the current activity partially conforms to the pattern, suggest the user with the likely (and possibly cheaper) alternative of the activity that is recorded in the pattern.

Figure 2. Suggestion Generator Algorithm

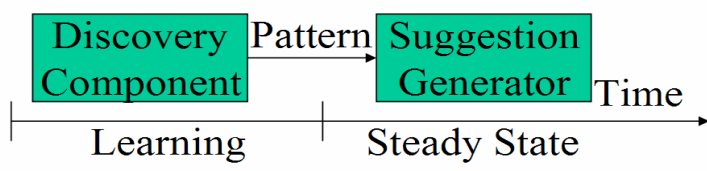

Figure 3. Operation

\section{Operation}

After the feature is installed, the discovery component discovers the patterns during the learning phase and seeds the prediction module, which starts predicting after the learning phase elapses as shown in Figure 3. The discovery component would keep running even after the Learning phase, and provide new patterns to the Suggestion Generator if there is a significant change in the usage pattern over time. The Suggestion Generator would be able to provide meaningful suggestions only after the Learning Phase.

\section{EXPERIMENTS}

We collected data from 4 users from the lower income group in and around Bangalore. Further to that, we collected the usage logs of people from the more well-to-do group too. We split the logs into two sets; the training set was fed into the pattern discovery algorithm followed by initiation of the suggestion generator with the test set. Firstly, we evaluate the hypothesis of the existence of a strong usage pattern from the lower income group by running the discovery algorithm. Secondly, as the test set also is expected to follow the same pattern as the training set, the comparison of the suggested durations of calls against the actual durations of the calls made would suggest how effectively the model was adhered to, by means of the suggestions. Thirdly, we evaluate the sensitivity of the suggestion generator to deviations. In a real-world scenario, the effectiveness of the suggestions would be quantified by the amount of cost savings (or the number of budget overshoots avoided) achieved by means of the suggestions generated. We expect that the measures detailed above approximate the on-field effectiveness measure.

\section{Pattern Discovery}

We analyzed the logs of both the lower income group and the well-to-do group and found that the daily pattern is much stronger than any pattern of a shorter duration. It may be noted that the discovery module is interested in finding out the smallest duration for which there exists a strong pattern. Further, the lower income group has a much stronger daily pattern than the latter. Table 1 lists the results of the entropy analysis. It may be noted that lesser the entropy, stronger the pattern. The confidence in the daily pattern is found to be much more than the confidence in a half-day pattern.

\begin{tabular}{|l|l|l|}
\hline Group & Daily Entropy & Half-Daily Entropy \\
\hline Lower Income & 3.50 & 4.19 \\
\hline Well-To-Do & 4.12 & 4.98 \\
\hline
\end{tabular}

Table 1. Pattern Discovery Results

\begin{tabular}{|l|l|}
\hline Training Data Size & Distance \\
\hline 1 week & 2.64 \\
\hline 2 weeks & 0.73 \\
\hline 3 weeks & 0.55 \\
\hline
\end{tabular}

Table 2. Pattern Adherence Analysis

\section{Pattern Adherence}

To evaluate whether the suggestion generator generates suggestions in tune with the assumed pattern (as is the desirable case), we did an analysis of duration suggestions. As the test set is also from the same model, the proximity of the suggested duration to the actual duration is a measure of model adherence. We measure the distance as the average number of standard deviations that the suggested duration is 
away, from the actual duration. The lesser the difference, the stronger the pattern adherence. We report the distances for varying amounts of training data for the lower income group in Table 2. As can be seen, the suggested durations approach the actual ones with sufficient training data.

\section{Deviation Sensitivity Analysis}

Although the technique has been found to be effective in suggesting strong adherence to the pattern used, the utility of the technique largely stems from its ability to detect deviations. We evaluate the deviation detection sensitivity of the algorithm by feeding the suggestion generator with two sets of entries; the pure test set of 20 entries and the test set randomly injected with 5 spurious out-of-pattern entries. As the prediction depends on the past history of calls made (which may include spurious calls if they exist), isolating the effect of spurious entries is non-trivial. The effect of spurious patterns in derailing the suggested durations is a measure of deviation sensitivity. We found that the average distance of suggested durations to the actual durations measured as the number of standard deviations slipped from 0.55 (for the pure test set) to 0.79 with the introduction of spurious entries. This suggests that the suggestion generator is able to detect the deviations from the pattern caused by the introduction of spurious entries.

\section{Implementation Feasibility}

A challenge in implementing learning systems on mobile phones is the limited memory and processing power available on the device. In our approach, the discovery module would require ' $n$ ' previous entries (this can correspond to a time window) of the usage history log for discovering the patterns. Many mobile devices store fixed number of $\log$ entries and multiples of $n$ can be stored dependent on the mobile storage. Further, storage for $\mathrm{n}$ can be reduced by having to store only representative samples from the log. For the suggestion generation component, the storage required is just the space required to store the usage pattern and the usage logs so far in the duration instance.

The clustering algorithm is run once in a fixed time window to discover the pattern. Thus it runs once in a regular interval, can be scheduled to run at low-load times and does not require processing in "real-time". The suggestion generation component is the "real-time" component would require comparing an activity with the usage pattern. Since the attributes of a pattern are very limited, the comparison would take trivial power which the current technology in mobile phones could very well accommodate.

\section{CONCLUSIONS AND FUTURE WORK}

In recent years, there has been an increasing trend in enabling the mobile device with additional features; especially those features the users would make use of on a daily basis. These include the radio, calculator, games, media player, camera, storage etc. Interface innovations particularly targeting the lower income group include introduction of the flashlight on the mobile device $[6,7]$.
Although more and more responsibilities are being delegated to the mobile device, usage cost control, which is the prime concern of the mobile user in the lower income group [2,3] is still left to the mobile user to perform manually. This work presents a first step towards interface innovations to enable the mobile user to delegate usage cost control tasks to the mobile. We show that an approach which exploits patterns in mobile usage would lead to fairly useful cost control suggestions, ensuring that the user would adhere to his usage pattern so that there would not be frequent overshoots in budget.

Although exploiting patterns in mobile usage is a promising approach to automate mobile usage cost, there could be more kinds of inputs that could enable the automatic cost control component to work better. There could be ways other than pro-active suggestions towards controlling cost. At times, it could well turn out that there is a cheaper alternative to a recurring activity which may have to be pointed out to the user; this would amount to deviating from the model, but for good. Exploring such alternative means of cost control would be an interesting future work towards the broad goal of empowering the mobile device with routine tasks undertaken by people in the lower strata of the society; thereby enabling better mobile penetration.

\section{ACKNOWLEDGEMENT}

We thank Karthik Jayakumar for his assistance in helping us to communicate with the people in Bangalore without which, this work would not have been possible.

\section{REFERENCES}

1. India's Evolving Mobile Market and Comparisons with China. http://www.ptc.org/events/ptc07/program/papers/T13_V iratPatel.pdf.

2. Jhunjhunwala, A., Can Telecom and IT be for the Disadvantaged? http://www.tenet.res.in/Papers/TelIT/TelecomAndIT.html

3. Blixt, P., Mobile Telephony in Rural India. Adapting the mobile telephone to the conditions of the unprivileged rural India, Master's Thesis, Royal Inst. of Tech., Sweden, 2005.

4. Deepak P, Bhamidipaty, A., Optimizing on Mobile Usage Cost for the Lower Income Group: Insights and Recommendations. Proc. Interact 2007, 339-342.

5. Abraham, R., Mobile Phones and Economic Development: Evidences from the Fishing Industry in India, Proc. ICTD 2006, 48-56.

6. R Bhagat, Rural India Calls, The Hindu, September 2007,http://www.thehindubusinessline.com/life/2007/09 /21/ stories/2007092150010100.htm

7. Cellphone Makers Focus on Rural Areas, China Daily, July 2007 , http://www.china.org.cn/english/BAT/215821.htm 